\title{
An online daily diary study of alcohol use using Amazon's Mechanical Turk
}

\author{
MARCELLA H. BOYNTON ${ }^{1}$ \& LAURA SMART RICHMAN ${ }^{2}$ \\ ${ }^{1}$ Lineberger Comprehensive Cancer Center, University of North Carolina, Chapel Hill, USA, and ${ }^{2}$ Department of \\ Psychology, Duke University, Durham, USA
}

\begin{abstract}
Introduction and Aims. In recent years, unprecedented levels of Internet access and the widespread growth of emergent communication technologies have resulted in significantly greater population access for substance use researchers. Despite the research potential of such technologies, the use of the Internet to recruit individuals for participation in event-level research has been limited. The purpose of this paper is to provide a brief account of the methods and results from an online daily diary study of alcohol use. Design and Methods. Participants were recruited using Amazon's Mechanical Turk. Eligible participants completed a brief screener assessing demographics and health behaviours, with a subset of individuals subsequently recruited to participate in a 2 week daily diary study of alcohol use. Results. Multilevel models of the daily alcohol data derived from the Mechanical Turk sample $(\mathrm{n}=369)$ replicated several findings commonly reported in daily diary studies of alcohol use. Discussion and Conclusions. Results demonstrate that online participant recruitment and survey administration can be a fruitful method for conducting daily diary alcohol research. [Boynton $\mathrm{MH}$, Richman LS. An online daily diary study of alcohol use using Amazon's Mechanical Turk. Drug Alcohol Rev 2014]
\end{abstract}

Key words: alcohol, daily diary, Mechanical Turk, MTurk.

\section{Introduction}

Obtaining accurate measures of alcohol consumption using traditional survey measures can be challenging due to retrospective biases as well as to substantial within- and between-person variations in consumption patterns $[1,2]$. Daily diary survey methodology, defined as the administration of a brief daily survey for a series of weeks, offers a great potential for assessing alcohol use proximate to its real-time occurrence $[3,4]$. Past research has employed a variety of technologies to collect daily measures of alcohol [5-9]; however, to date, there have been a dearth of studies leveraging online recruitment platforms for the purposes of daily diary research, in general, and alcohol-related research, specifically.

Amazon's Mechanical Turk (MTurk) is an online crowdsourcing tool that allows 'workers' to complete online tasks or 'human intelligence tasks' (HITS) for relatively small amounts of remuneration. MTurk has become an increasingly popular tool for social science research, with multiple experimental and survey studies consistently replicating findings from prior research [10-24]. The purpose of this paper is to illustrate the utility of MTurk to recruit a diverse sample of adults for participation in an online daily diary study of alcohol use.

\section{Methods \\ Participants}

Five hundred and eighteen participants enrolled in the daily diary study and completed at least one daily survey. Of those, 130 were removed because they completed fewer than four daily surveys, and an additional 19 were removed because they did not have at least two consecutive daily surveys. The final sample was

Marcella H. Boynton PhD, Research Scientist, Laura S. Richman PhD, Assistant Professor. Correspondence to Dr Marcella H. Boynton, Lineberger Comprehensive Cancer Center, University of North Carolina, 450 West Drive, Chapel Hill, NC 27514, USA. Tel: +1 (919) 966 2759; Fax: +1 (919) 843 3418; E-mail: mhb23@unc.edu 
comprised of 369 adults contributing 3145 daily observations. The average number of completed daily measures was 8.5 [standard deviation $(\mathrm{SD})=3.9$ ] . Table 1 presents general sample demographics.

\section{Procedure}

Participants were recruited as part of a larger online survey study of personality and health that was comprised of three separate recruitment phases. Initially, we posted a HIT on MTurk inviting interested individuals to complete a brief screener assessing basic demographic and health factors. Inclusion criteria for the follow-up survey were a primary racial/ethnic identity of White, Black/African American, Latino or Asian/ Pacific Islander, age 21-65, currently residing in the United States (US), and ability to speak and read English. Because one of the broader aims of the study was focused on issues particularly relevant to US ethnic minority groups, an over-sampling approach was employed such that enrolment for each of the four targeted ethnic groups was kept relatively balanced. Individuals invited to complete the follow-up survey were sent a unique link to a secure website [25] where they completed a series of social-personality and health-related measures. Participants recruited for the daily diary study used the same secure website to com- plete a 5-min daily survey for up to 14 consecutive days. Each daily survey assessed cognitions and behaviours for the prior evening (5 PM to $6 \mathrm{AM}$ ) and the current day (6 AM to $5 \mathrm{PM}$ ) and could be completed between 5:00 PM (at which time an email reminder was sent to participants) and 6:00 AM the following morning. Participants were compensated for completing both the screener/baseline survey (\$US 0.85) and daily diary surveys (up to \$US 5.00 for perfect adherence).

\section{Measures}

Possible history of alcohol misuse was assessed using the four-item CAGE Alcohol Questionnaire [26,27]; concordant with past research $[28,29]$, the CAGE was coded 0 for zero item endorsement and 1 for $\geq 1$ item(s) endorsed. Depression was assessed using the eight-item Center for Epidemiologic Studies Depression (CES-D) Scale [30], which is a validated short version of the 20-item CES-D [31]. Both the eight-item CES-D $(\alpha=0.78)$ and the four-item CAGE $(\alpha=0.74)$ exhibited an acceptable scale reliability in our sample.

In order to maximise the reliability of self-reported daily alcohol consumption, a figure with accompanying text depicting a 'standard drink' for each major type of alcohol was presented immediately prior to the daily alcohol measures (see Figure 1). When reporting daily

Table 1. Descriptive statistics

\begin{tabular}{|c|c|c|}
\hline & $\begin{array}{l}\text { No history of alcohol misuse } \\
\text { CAGE }=0 ; n=297\end{array}$ & $\begin{array}{l}\text { Possible history of alcohol } \\
\text { misuse CAGE } \geq 1 ; n=71\end{array}$ \\
\hline Baseline measures & $M(S D)$ or $\%$ & $M(S D)$ or $\%$ \\
\hline \multicolumn{3}{|l|}{ Gender } \\
\hline$\%$ Male & 42.8 & 49.3 \\
\hline$\%$ Female & 57.2 & 50.7 \\
\hline Age & $31.5(9.4)$ & $31.8(10.1)$ \\
\hline \multicolumn{3}{|l|}{ Race } \\
\hline \% White/Caucasian & 28.0 & 35.2 \\
\hline$\%$ Black/African American & 27.6 & 31.0 \\
\hline$\%$ Latino/Hispanic & 17.5 & 16.9 \\
\hline$\%$ Asian/Pacific Islander & 26.9 & 16.9 \\
\hline Income bracket & $\$ 55000-59999(\$ 40000)$ & $\$ 50000-54999(\$ 40000)$ \\
\hline \multicolumn{3}{|l|}{ Education } \\
\hline \% High school diploma or less & 11.1 & 9.9 \\
\hline \% Some college or associate's degree & 33.0 & 42.3 \\
\hline$\%$ College degree & 40.4 & 35.2 \\
\hline$\%$ Master's or doctoral-level degree & 15.5 & 12.6 \\
\hline CES-D Scale & $6.8(4.4)$ & $7.7(4.3)$ \\
\hline \multicolumn{3}{|l|}{ Daily measures } \\
\hline Number of completed daily surveys & $7.24(3.77)$ & $8.81^{\star}(3.83)$ \\
\hline Number of reported drinking days & $1.38(2.34)$ & $2.68^{\star}(2.91)$ \\
\hline Number of reported heavy drinking days & $0.26(0.91)$ & $1.18^{\star}(2.18)$ \\
\hline
\end{tabular}

${ }^{\star}$ significantly higher mean or median, $P<0.05$. CES-D, Center for Epidemiologic Studies Depression; SD, standard deviation. 


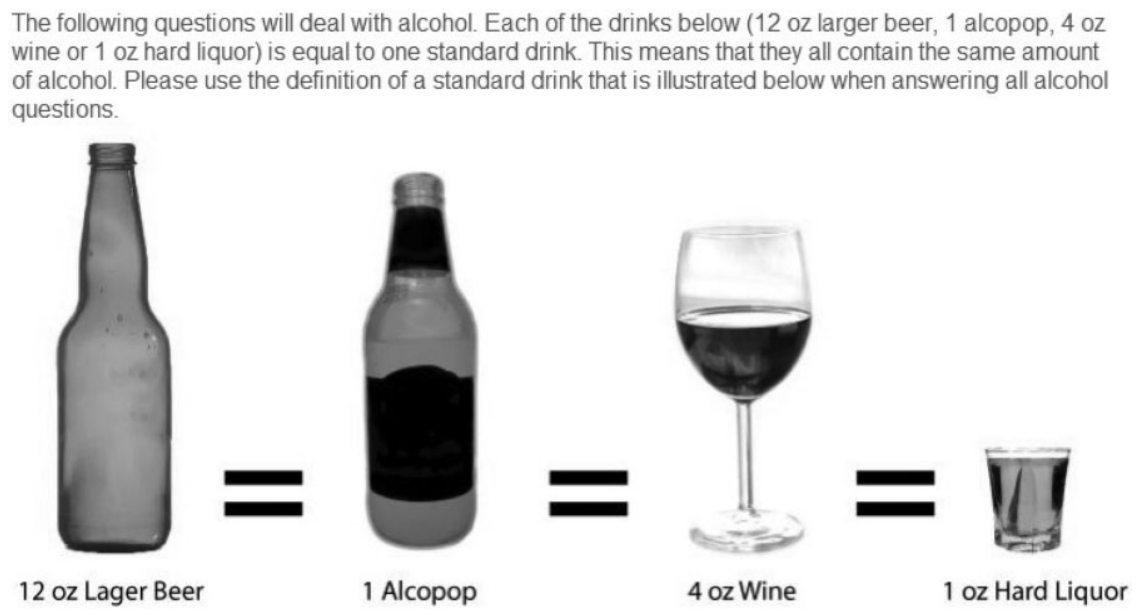

Figure 1. Alcohol measures introduction and standard drink definition (note: 1 oz is equal to $28.4 \mathrm{~g}$ ).

alcohol consumption, individuals were asked to specify type and number of drinks consumed, again with accompanying imagery (see Figure 2). Alcohol use intentions were measured using the item 'Approximately how many standard alcoholic drinks do you intend to consume between $5 \mathrm{PM}$ today and $5 \mathrm{PM}$ tomorrow?' Alcohol salience was measured with the item 'How much have you thought about alcohol or alcohol-related places and activities since waking up this morning?'

\section{Results}

Of the 369 participants, $87 \%(n=322)$ reported some level of past alcohol consumption. The average number of drinking days and heavy drinking days across the entire sample was $1.6(\mathrm{SD}=2.5)$ and $0.45(\mathrm{SD}=1.3)$, respectively. Those with a positive CAGE score had a significantly higher number of drinking days $(M=2.7$; $\mathrm{SD}=2.9)$ and heavy drinking days $(M=1.2 ; \mathrm{SD}=2.2)$ compared with those without a positive CAGE score $(M=1.4 ; \mathrm{SD}=2.4 ; M=0.3 ; \mathrm{SD}=0.9$, respectively $), F$ $(1,366)=15.9, P<0.001$ for drinking days and $F(1$, $366)=30.7, P<0.001$ for heavy drinking days.

Of the 3145 reported days, approximately 19\% $(n=600)$ included the consumption of one or more standard alcoholic drinks. Of those, 36\% $(n=214)$ were beer only, $20 \%$ were wine only $(n=122), 9 \%$ were shots only $(n=54), 11 \%$ were mixed drinks only $(n=64), 3 \%$ were some other form of liquor (e.g., alcopop, malt liquor, $n=18$ ) and $21 \%$ were two or more different types of alcoholic beverages $(n=128)$. Of the 600 reported drinking days, $27 \%(n=162)$ met the National Institute on Alcohol Abuse and Alcoholism (NIAAA) criteria for a heavy drinking episode, which is $\geq 4$ drinks for females and $\geq 5$ for males [32]. The average number of drinks consumed when drink- ing occurred was $3.6(\mathrm{SD}=3.5)$. Those who reported at least one drinking day during the study had a slightly higher measures completion rate $(M=9.0, \mathrm{SD}=3.4)$ compared with those who reported no drinking $(M=8.5, \quad \mathrm{SD}=3.9), \quad F \quad(1,367)=4.45, P=0.01$. However, those with a history of problematic drinking, as measured by the CAGE, had a slightly lower measures completion rate $(M=7.2, \mathrm{SD}=3.8)$ compared with those who reported no problematic history drinking $(M=8.8, \mathrm{SD}=3.8), F(1,366)=9.74, P=0.002$.

Multilevel modelling was used to analyse the daily diary data. Number of drinks consumed (0-15+) and heavy drinking episode $(0=n o ; 1=y e s)$ were the two daily alcohol outcomes of interest. Random intercept models were estimated using the PROC GLIMMIX procedure in SAS 9.3 [33]. Table 2 details all model results. In the final combined effects model for number of drinks consumed, being male, having a history of problematic drinking, daily alcohol intentions, daily alcohol salience and weekend day predicted a higher number of drinks consumed. For the heavy drinking episode final combined effects model, odds of having a heavy drinking day was greater for those individuals who were Latino (as compared with White), had a history of problematic drinking, or reported alcohol intentions or salience at the daily level.

\section{Discussion}

One of the primary purposes of this study was to develop and successfully implement a daily diary study of alcohol using MTurk to recruit a diverse US sample. Examination of the measures adherence rates and demographic factors indicates that we were successful in this regard. Further, our alcohol-related findings replicated a number of associations noted in previous research. In particular, daily alcohol consumption was 
Please enter the approximate number of each type of standard alcoholic drinks you consumed:

\title{
LAST NIGHT \\ (from 5 PM to 6 AM)
}

\&

TODAY

(from 6 AM to 5 PM)

\begin{abstract}
For example, if you drank two $12 \mathrm{oz}$ cans of Bud Lite last night around 9 PM, then you would enter a 2 in the top left box. You can use the "Tab" button to quickly move from box to box. Please enter 0 for each listed beverage that you did not consume.
\end{abstract}
\# of standard drinks consumed \# of standard drinks consumed LAST NIGHT
between $5 \mathrm{PM}$ to $6 \mathrm{AM}$
TODAY
between $5 \mathrm{PM}$ to $6 \mathrm{AM}$
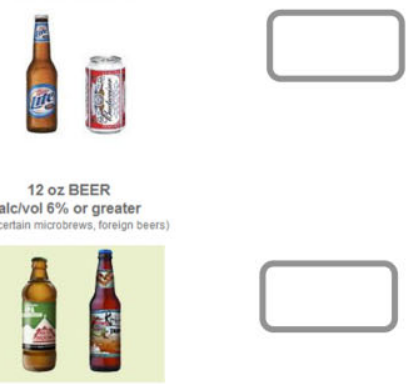

4 oz WINE or CHAMPAGNE
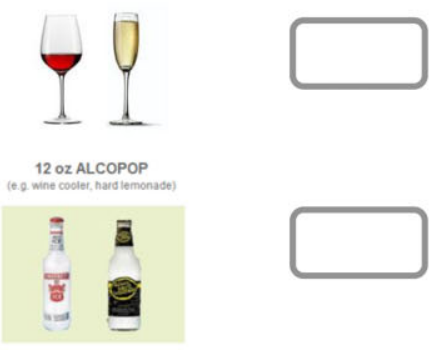
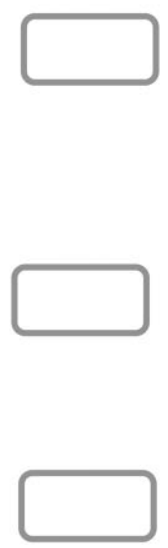
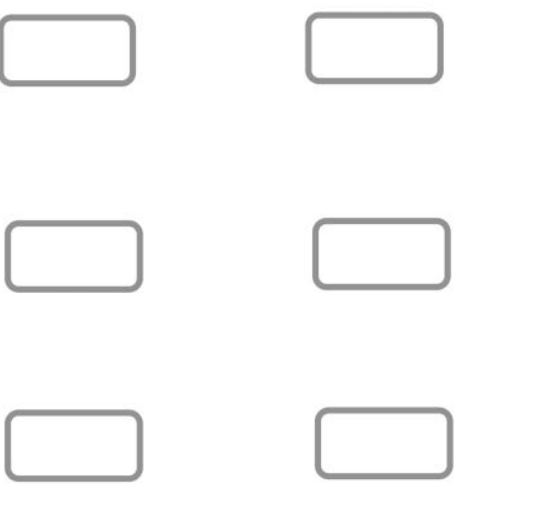

1 shot of HARD LIQUOR

(eg. whisker, tequila)
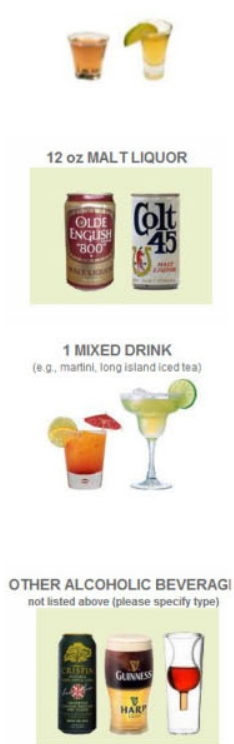

\# of standard drinks consumed \# of standard drinks consumed LAST NIGHT TODAY

between 5 PM to 6 AM between 5 PM to 6 AM

Figure 2. Daily alcohol consumption measures (note: 1 oz is equal to $28.4 \mathrm{~g}$ ).

greater on weekends, for those with a positive CAGE score, and when alcohol salience and intentions were present. Reflective of typical US drinking preferences and behaviours [34], beer was the most common type of beverage consumed, followed by various forms of spirits and liquors, and then wine was the least common type of alcohol consumed.

\section{Limitations and future directions}

Although our findings were very similar to those described in past daily dairy studies of alcohol, it should be noted that there were several key demographic differences, with our sample substantially older, more affluent and more ethnically diverse than a typical college student sample. Another major difference between this and past samples is that we did not require a certain level of past alcohol use for participation. Researchers interested in studying patterns of alcohol use in groups more similar to those in past daily diary studies could readily adapt MTurk screening criteria to select for such individuals, and future online daily diary alcohol studies would benefit from this approach. Also of note, daily measures adherence in our study was approximately $60 \%$, which is somewhat lower than the typical $75-85 \%$ rates found in many college student daily diary studies of alcohol use. One contributing factor may be the level of compensation, which was a 
Table 2. Predicting number of drinks and heavy drinking episodes on the daily level

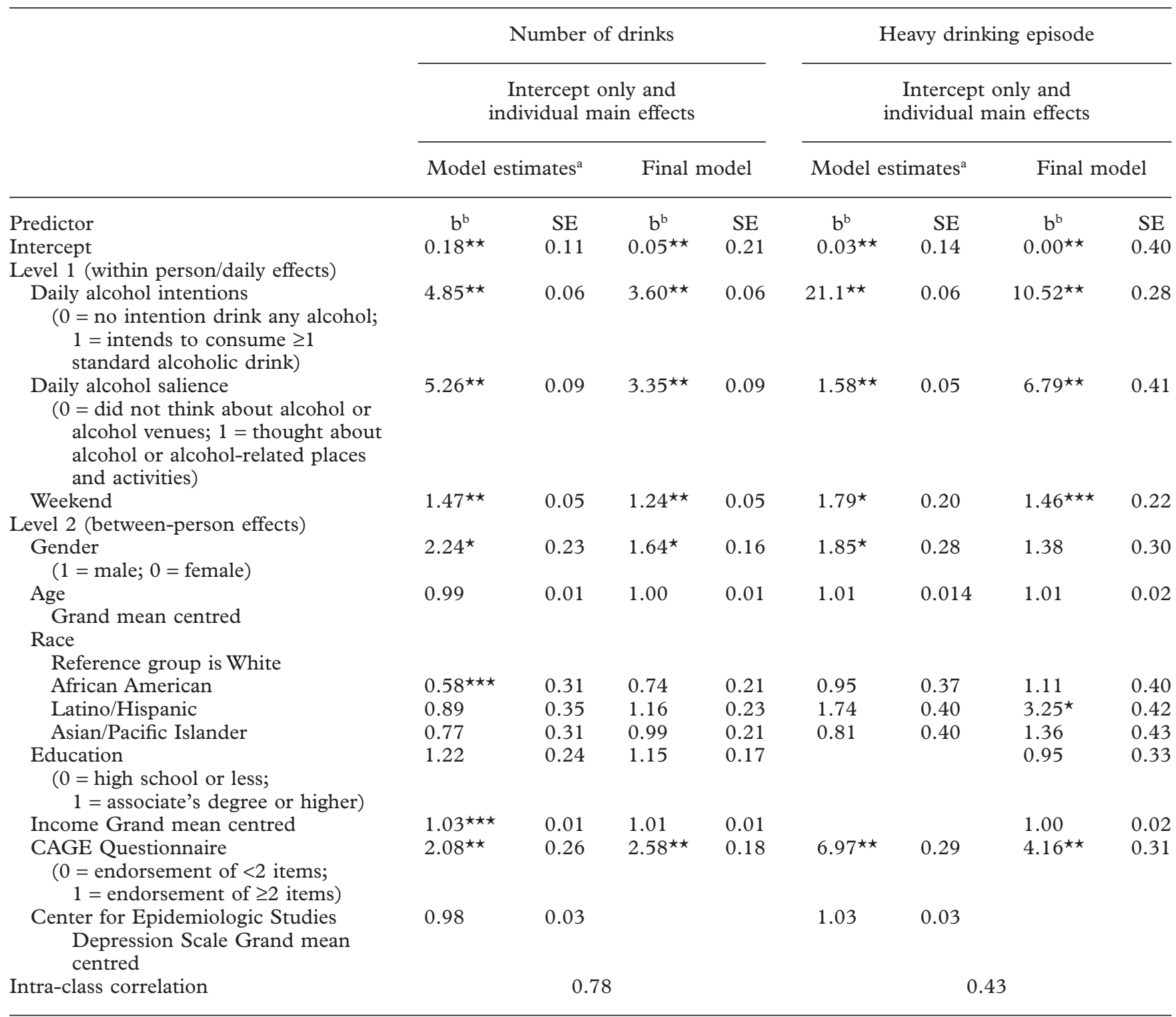

${ }^{\star} P<0.05 ;{ }^{\star \star} P<0.0001 ;{ }^{\star \star \star} P<0.10$ (marginally significant). ${ }^{a}$ Each estimate in this column is unadjusted for all other predictors; the final model column presents the adjusted estimates from the combined effects model; ${ }^{b}$ Estimates provided are exponentiated betas to reflect the true drink number estimate or odds ratio. SE, standard error.

small fraction of what is typically offered. Future online daily diary research adherence rates would likely benefit from a higher incentive schedule. In summary, this research demonstrates the potential value of using online participant recruitment for daily diary alcohol research.

\section{Acknowledgements}

This research was supported by National Institute on Drug Abuse Grant P30 DA023026, and manuscript preparation was supported, in part, by National
Institutes of Health Grants R21 AA017584, M01RR10284, UL1RR031975 and T32AA007290. Its contents are solely the responsibility of the authors and do not necessarily represent the official views of the National Institutes of Health or National Institute on Drug Abuse. The authors extend their gratitude to Michael Asher for Web programming and implementation of the study, Anna Wise for study and database management, Micah Lattanner and Rick Hoyle for study design feedback, and Steve Armeli and Howard Tennen for their comments on a version of this manuscript. 


\section{References}

[1] Fitzgerald JL, Mulford HA. Self-report validity issues. J Stud Alc. 1987;48:207-11.

[2] Stockwell T, Donath S, Cooper-Stanbury M, Chikritzhs T, Catalano P, Mateo C. Under-reporting of alcohol consumption in household surveys: a comparison of quantityfrequency, graduated-frequency and recent recall. Addiction 2004;99:1024-33.

[3] Armeli S, Todd M, Mohr CA. Daily process approach to individual differences in stress-related alcohol use. J Pers 2005;73:1657-86.

[4] Conner TS, Tennen H, Fleeson W, Barrett LF. Experience sampling methods: a modern idiographic approach to personality research. Soc Personal Psychol Compass. 2009; 3:292-313.

[5] Armeli S, Carney MA, Tennen H, Affleck G, O’Neil TP. Stress and alcohol use: a daily process examination of the stressor-vulnerability model. J Pers Soc Psychol 2000; 78:979-94.

[6] Mohr CD, Armeli S, Tennen H, Carney MA, Affleck G, Hromi A. Daily interpersonal experiences, context, and alcohol consumption: crying in your beer and toasting good times. J Pers Soc Psychol 2001;80:489-500.

[7] Barrett LF, Barrett DJ. An introduction to computerized experience sampling in psychology. Soc Sci Comp Rev. 2001;19:175-85.

[8] Sacco P, Smith CA, Harrington D, Svoboda DV, Resnick B. Feasibility and utility of experience sampling to assess alcohol consumption among older adults. J Appl Gerontol 2014. Epub ahead of print.

[9] O'Hara RE, Boynton MH, Scott D, et al. Drinking to cope among African-American college students: an assessment of episode-specific motives. Psychol Addict Behav in press.

[10] Azzam T, Jacobson MR. Finding a comparison group: is online crowdsourcing a viable option? Amer Jour Eval. 2013;34:372-84.

[11] Amir O, Rand DG, Gal YK. Economic games on the internet: the effect of $\$ 1$ stakes. PLoS ONE 2012;7: e31461.

[12] Casler K, Bickel L, Hackett E. Separate but equal? A comparison of participants and data gathered via Amazon's MTurk, social media, and face-to-face behavioral testing. Comput Human Behav 2013;29:2156-60.

[13] Gardner RM, Brown DL, Boice R. Using Amazon's Mechanical Turk website to measure accuracy of body size estimation and body dissatisfaction. Body Image 2012; 9:532-4.

[14] Holden CJ, Dennie T, Hicks AD. Assessing the reliability of the M5-120 on Amazon's Mechanical Turk. Comput Human Behav 2013;29:1749-54.

[15] Horton J, Rand D, Zeckhauser R. The online laboratory: conducting experiments in a real labor market. Exp Econ. 2011;14:399-425.

[16] Rand DG. The promise of Mechanical Turk: how online labor markets can help theorists run behavioral experiments. J Theor Biol 2012;299:172-9.
[17] Simons DJ, Chabris CF. Common (mis)beliefs about memory: a replication and comparison of telephone and mechanical turk survey methods. PLoS ONE 2012; 7:e51876.

[18] Summerville A, Chartier CR. Pseudo-dyadic 'interaction' on Amazon's Mechanical Turk. Behav Res Methods 2013;45:116-24.

[19] Joinson A. Social desirability, anonymity, and internetbased questionnaires. Behav Res Meth Instrum Comput. 1999;31:433-8.

[20] Gosling SD, Vazire S, Srivastava S, John OP. Should we trust web-based studies? A comparative analysis of six preconceptions about internet questionnaires. Am Psychol 2004;59:93-104.

[21] Berinsky AJ, Huber GA, Lenz GS. Evaluating online labor markets for experimental research: Amazon.com's Mechanical Turk. Pol Anal. 2012;20:351-68.

[22] Paolacci G, Chandler J, Ipeirotis PG. Running experiments on Amazon Mechanical Turk. Judgm Decis Mak. 2010; 5:411-19.

[23] Buhrmester M, Kwang T, Gosling SD. Amazon's MechanicalTurk: a new source of inexpensive, yet high-quality, data? Perspect Psychol Sci. 2011;6:3-5.

[24] Mason W, Suri S. Conducting behavioral research on Amazon's Mechanical Turk. Behav Res Methods 2012; 44:1-23.

[25] Qualtrics (2012). Qualtrics, Provo, Utah. Available at: http://www.qualtrics.com (accessed May 2014).

[26] Ewing JA. Detecting alcoholism: the CAGE questionnaire. JAMA 1984;252:1905-7.

[27] O'Brien CP. The CAGE questionnaire for detection of alcoholism: a remarkably useful but simple tool. JAMA 2008;300:2054-6.

[28] Bush B, Shaw S, Cleary P, Delbanco TL, Aronson MD. Screening for alcohol abuse using the CAGE questionnaire. Am J Med 1987;82:231-5.

[29] Girela E, Villanueva E, Hernandez-Cueto C, Luna JD. Comparison of the CAGE questionnaire versus some biochemical markers in the diagnosis of alcoholism. Alcohol Alcohol 1994;29:337-43.

[30] Andresen EM, Byers K, Friary J, Kosloski K, Montgomery R. Performance of the 10-item Center for Epidemiologic Studies Depression scale for caregiving research. SAGE Open Med. 2013;1:1-9.

[31] Radloff LS. The CES-D scale: a self-report depression scale for research in the general population. Appl Psych Meas. 1977;1:385-401.

[32] National Institute on Alcohol Abuse and Alcoholism, NIAAA. NIAAA council approves definition of binge drinking. Washington, DC: Office of Research Translation and Communication, 2004. NIAAA Newsletter, 3, 3, Winter.

[33] SAS Institute Inc. Base $S A S \circledR 9.3$ procedures guide. Cary, NC: SAS Institute Inc., 2011.

[34] National Institute on Alcohol Abuse and Alcoholism, NIAAA (2009). Apparent per capita alcohol consumption: National, state, and regional trends, 1977-2009. Available at: http://pubs.niaaa.nih.gov/publications/Surveillance92/ CONS09.pdf (accessed May 2014). 